\title{
Décadrages Décadrages
}

cinéma, à travers champs Cinéma, à travers champs

29-30 | 2015

René Vautier

\section{Taiyo Onorato et Nico Krebs à Art|Basel 2014 : entre imaginaire mondialisé et « territoire géopolitique} sans image »

\section{Claus Gunti}

\section{(2) OpenEdition}

Journals

Édition électronique

URL : https://journals.openedition.org/decadrages/818

DOI : $10.4000 /$ decadrages. 818

ISSN : 2297-5977

Éditeur

Association Décadrages

Édition imprimée

Date de publication : 1 avril 2015

Pagination : 239-249

ISBN : 9782970096306

ISSN : 2235-7823

Référence électronique

Claus Gunti, «Taiyo Onorato et Nico Krebs à Art|Basel 2014 : entre imaginaire mondialisé et « territoire géopolitique sans image » », Décadrages [En ligne], 29-30 | 2015, mis en ligne le 01 mars 2017, consulté le 24 mars 2023. URL : http://journals.openedition.org/decadrages/818 ; DOI : https:// doi.org/10.4000/decadrages. 818

\section{(c) (i) (2) (2)}

Creative Commons - Attribution - Pas d'Utilisation Commerciale - Partage dans les Mêmes Conditions 4.0 International - CC BY-NC-SA 4.0

https://creativecommons.org/licenses/by-nc-sa/4.0/ 


\section{Taiyo Onorato et Nico Krebs à Art|Basel 2014: entre imaginaire mondialisé et « territoire géopolitique sans image »}

Le projet de Taiyo Onorato et Nico Krebs, présenté dans la section «Statements» ${ }^{1}$ de la foire d'art contemporain Art|Basel en juin 2014 (fig. I), résulte d'un voyage de 17 ooo kilomètres qui a conduit les deux photographes de Zurich à Oulan-Bator, en Mongolie. Leur périple automobile s'inscrit dans une réflexion sur le statut de la représentation photographique dans un contexte culturel saturé d'images, et vise plus particulièrement à documenter «un territoire géopolitique sans image ${ }^{2}$. La série Eurasia exposée à Bâle se concentre sur les limites géographiques entre l'Europe et l'Asie - le duo a notamment parcouru l'Ukraine, la Géorgie, l’Azerbaïdjan, le Turkménistan, l’Ouzbékistan, le Kirghizstan, le Kazakhstan, la Russie et la Mongolie $^{3}$-, avec un intérêt particulier pour les anciennes républiques soviétiques, relativement peu connues en Occident. Elle interroge l'existence visuelle de ces entités politiques qui tentent de s'établir dans un monde globalisé, mais aborde également la question de la fonction de l'artiste qui raconte ou interroge son appréhension du réel.

Dans le travail d'Onorato et Krebs, la réflexion sur l'omniprésence d'images diffusées par l'industrie culturelle s'exprime à travers deux pôles antagonistes. Les images montrées à Art|Basel portent essentiellement sur d'anciens pays satellites de l'URSS, le Turkménistan et l’Azerbaïdjan en particulier. Elles s'inscrivent dans une analyse d'un territoire

1 Section dédiée à des artistes émergents.

2 Ce projet rappelle une entreprise très similaire, réalisée par Charles de Meaux et Philippe Parreno, dont est tirée cette expression. En 1999, les deux plasticiens réalisèrent le long métrage Le Pont du Trieur sur le Pamir, région méconnue et partagée entre le Tadjikistan, l'Afghanistan, la Chine et le Kirghizstan. Ils racontaient l'histoire d'un monde «fictif», imaginaire car on n'en connaissait à l'époque que très peu de représentations, «redonnant vie à une géographie par l'image». Le film recréait une réalité hybride à partir d'images d'archive et d'images fictives, oscillant en permanence entre formes documentaires (entretiens filmés d'un botaniste tadjik ou d'un cinéaste exilé) et composantes évoquant plutôt une production de science-fiction (musique électronique, longs plans fixes, approche contemplative, etc.), sans que l'on puisse déterminer avec certitude le statut de chacun de ces éléments. Mais contrairement à la série d'Onorato et Krebs, le film chapeauté par la maison de production Anna Sanders est «tourné» en grande partie à Paris. Voir à ce propos Charles de Meaux, entretien avec Pascale Cassagnau, dans Pascale Cassagnau, Future Amnesia - Enquêtes sur un troisième cinéma, Paris, Editions Isthme, 2007, p. 103 et Claus Gunti, «La réalité remise en question: le statut de l'image dans l'œuvre de Philippe Parreno», Décadrages. Cinéma, à travers champs, $\mathrm{n}^{\circ} \mathrm{I} 3$, 2008, p. 33 (disponible également sur http://decadrages.revues.org/512).

3 Voir communiqué de presse, galerie RaebervonStenglin, Zurich, Statements with Taiyo Onorato \& Nico Krebs, Art/Basel, Basel, 19.06.14 - 22.06.14, disponible sur http://raebervonstenglin. com/index/fairs/Art-Basel-20I4.html, consulté le 4 avril 2015 . 


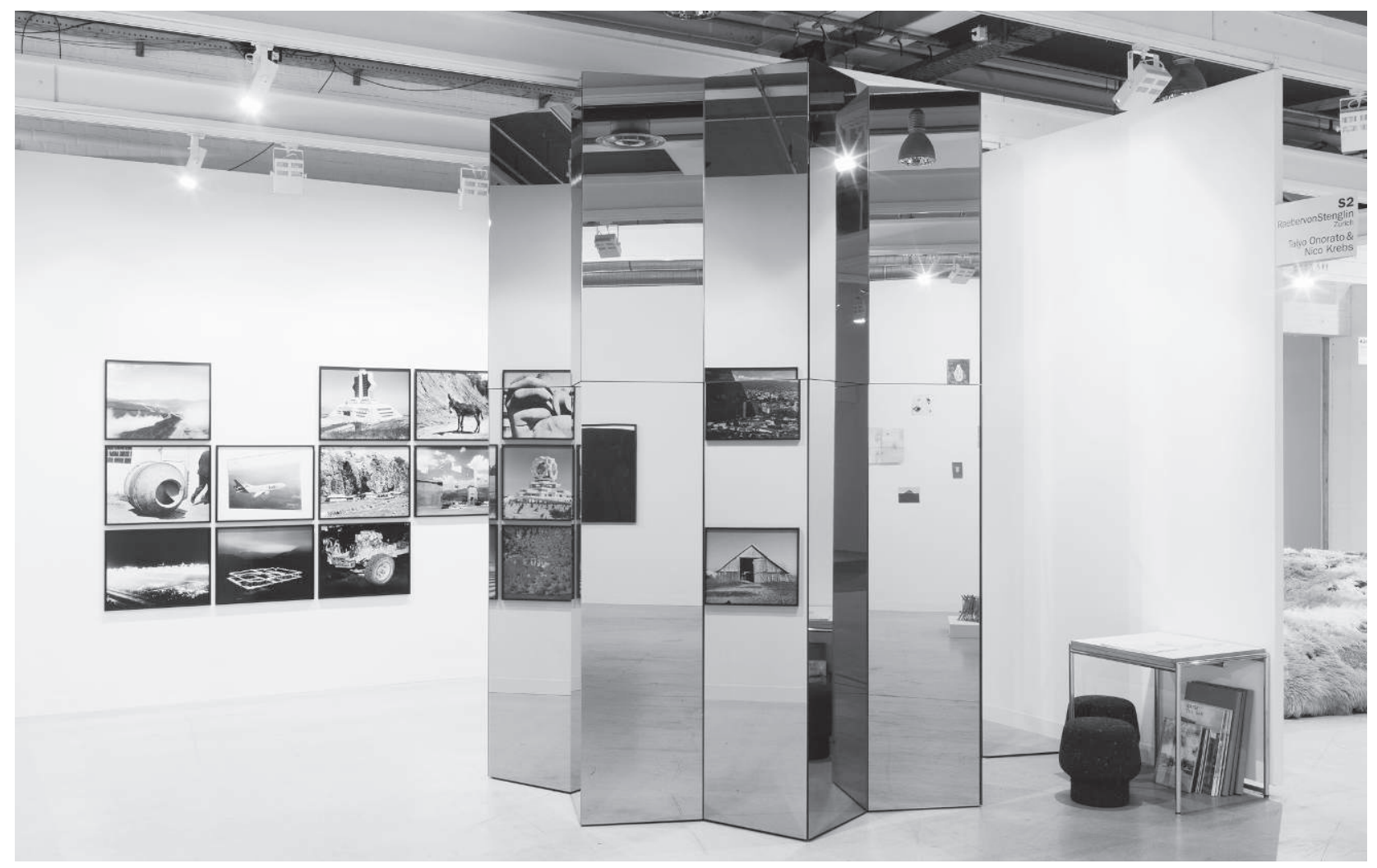

1/ Vue d'installation, stand de la galerie RaebervonStenglin, Statements, Art|Basel, 2014 (photographie Gunnar Meier; Courtesy Galerie RaebervonStenglin, Zurich)

4 Plus encore que la série elle-même, l'ouvrage figure déjà dans certaines anthologies des projets photographiques les plus importants des années 2000. Il est par exemple présenté dans le troisième volume de l'histoire du livre de photographie édité par Martin Parr et Jeffrey Badger, devenu l'une des références sur le sujet. Voir Taiyo Onorato et Nico Krebs, The Great Unreal, Zurich, Patrick Frey, 2008 et Martin Parr et Jeffrey Badger, The Photobook. A His-

sans images, ces pays n'ayant que peu investi la mémoire visuelle occidentale. The Great Unreal (2005-2008), la série de photographies et le livre d'artiste homonyme qui ont révélé les deux photographes ${ }^{4}$, incarnent quant à eux une proposition que l'on pourrait qualifier d'antinomique, puisqu'ils exposent le paysage surmédiatisé de l'Ouest américain ${ }^{5}$. Avant

tory. Volume 3, Londres, Phaidon, 2014, pp. 304-305.

5 Cette opposition entre paysages connus et paysages sans images a été for- mulée de manière explicite par Taiyo Onorato et Nico Krebs dernièrement, lors d'une conférence à l'Ecole cantonale d'art de Lausanne (écal), le ıo décembre 2014. 
d'analyser plus spécifiquement l'exposition bâloise, il est donc utile de résumer dans les grandes lignes les enjeux de ce travail, tant il concourt à appréhender la série provisoirement intitulée Eurasia ${ }^{6}$. Comme l’indique le titre de la série, la réalité dans The Great Unreal se trouve dès le départ mise en échec. Le projet, issu de plusieurs voyages à travers les Etats-Unis, répond à l'impossibilité de photographier l’Ouest américain, tant ce topos fut exploité au cours des $\mathrm{XIX}^{\mathrm{e}}$ et $\mathrm{XX}^{\mathrm{e}}$ siècles. Il procède par une mise en scène loufoque des images et des stratégies de reconstruction qui simulent l'aptitude reproductive du dispositif photographique, sapant tout ambition illusionniste. En intégrant des éléments insolites ( fig. 2), en manipulant la surface des images en laboratoire (fig.3) ou en reconstruisant des éléments de décor en carton afin de simuler - avec une recherche de réalisme toute relative - une photographie crédible ( fig. 4), Onorato et Krebs déconstruisent les paysages américains, tels que l'imaginaire collectif les restitue. Par différentes stratégies, ils en rejouent les codes et décomposent le médium photographique, les modifications parasitant le concept de transparence ou la lisibilité de l'image. Les deux artistes s'appuient par exemple sur des éléments humoristiques, qui permettent une appréhension plus distanciée du sujet représenté. En plaçant des frites devant le paysage majestueux du Grand Canyon, l'image mnémonique du regardeur de ce même motif se trouve écornée, produisant une sorte d'hilarité critique. A un niveau interprétatif, ces bricolages incongrus brouillent la lecture des images, les rendant énigmatiques (fig. 2) ou «sémantiquement» impossibles (fig. 4).

Cette stratégie loufoque n'est pas sans rappeler l'attitude de certains de leurs compatriotes, même si l'idée d'un art contemporain typiquement suisse se révèle méthodologiquement problématique. De nombreux curateurs, de Harald Szeemann à Gianni Jetzer, ont par ailleurs mis en œuvre des stratégies pour déjouer cette étiquette ${ }^{7}$. Toutefois, il est indéniable que ces bricolages saugrenus, mais réalisés avec beaucoup de soin, ce plaisir de la transgression et cette posture irrévérencieuse, rappellent la pratique de nombreux plasticiens suisses d'horizons divers à l'instar d'Olaf Breuning, qui partage avec Onorato et Krebs la réflexion critique sur la circulation des images associées à la culture populaire. Mais ce sont surtout Peter Fischli et David Weiss qui semblent logiquement s'imposer ici comme références. Cette «exploration des clichés avec un sérieux appliqué ${ }^{8}$ commence d'ailleurs à être
6 Le site internet des artistes propose ce titre générique pour la série en cours, dont Nico Krebs confirme le statut provisoire. En effet, les artistes sont retournés en Mongolie en 20I4 pour réaliser de nouvelles images; et s'ils ont montré publiquement certains rushs de films tournés en r6mm et quelques nouvelles photographies, le projet n'a pas encore de forme définitive. Discussion entre l'auteur et les artistes (Lausanne, décembre 20I4), correspondance e-mail avec Nico Krebs (8 mai 20I5) et http:// tonk.ch, consulté le is janvier 2015.

7 En particulier l'exposition Visionnäre Schweiz au Kunsthaus de Zurich (I99I) de Szeemann et A Sunday in the Mountains au Swiss Institute à New York (2013) de Jetzer. On trouvera par exemple chez Lionel Bovier, dans l'introduction du catalogueAcross/Art/Suisse/I975-200o, une synthèse des débats historiques et théoriques sur les limites de toute tentative de catégorisation allant dans ce sens. Lionel Bovier (éd.), Across/Art/ Suisse/I975-2000, Genève, Skira, 200I, pp. $7^{-15}$.

8 François Bovier, «La mécanique du rire dans les films de Peter Fischli et David Weiss », in Alain Boillat, Philipp Brunner, Barbara Flückiger (éd.), Cinéma $\mathrm{CH}$ : réception, esthétique, histoire, Schüren Verlag, Marburg, 2008, p. I64. 


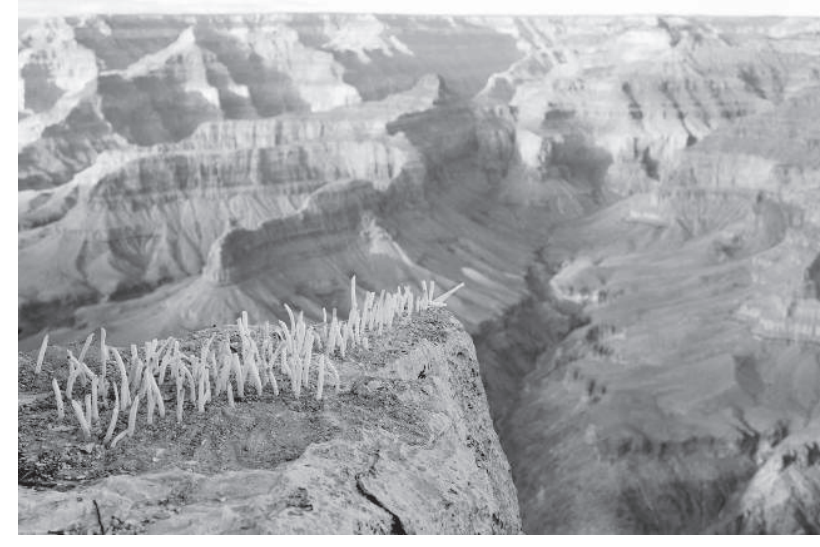

2/ Taiyo Onorato \& Nico Krebs, Pommes Frittes, 2005 (c-print, $54 \times 70 \mathrm{~cm}$, ( ) Taiyo Onorato \& Nico Krebs; Courtesy the artists and Galerie RaebervonStenglin, Zurich)

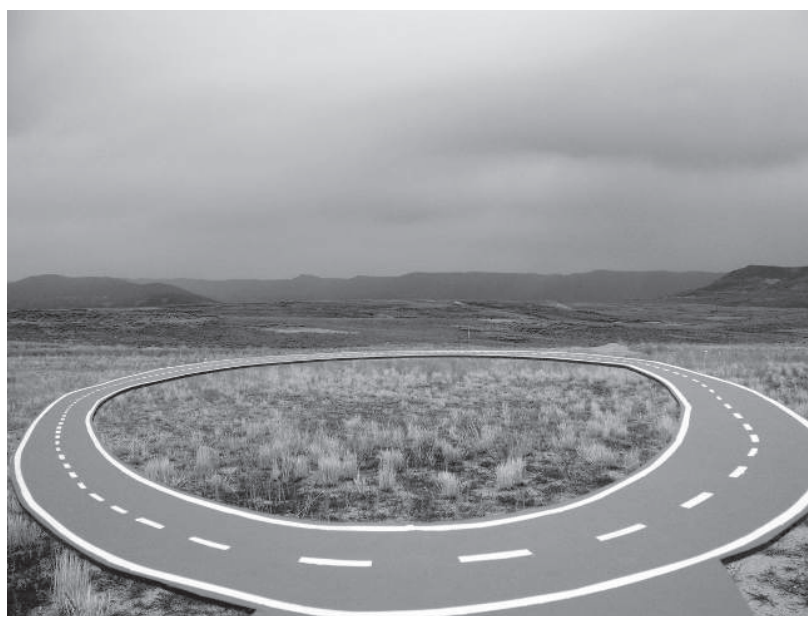

4/ Taiyo Onorato \& Nico Krebs, Happy Ending, 2005 (c-print, $18 \times 23 \mathrm{~cm}$, (c) Taiyo Onorato \& Nico Krebs; Courtesy the artists and Galerie RaebervonStenglin, Zurich)

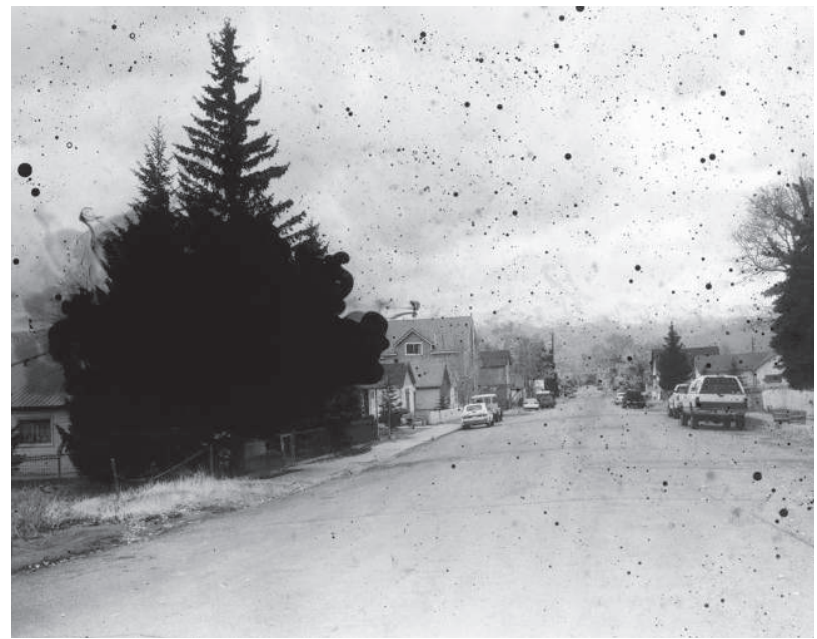

3/ Taiyo Onorato \& Nico Krebs, Leadville 2, 2008 (c-print, $50 \times 65 \mathrm{~cm}$, (c) Taiyo Onorato \& Nico Krebs; Courtesy the artists and Galerie RaebervonStenglin, Zurich)
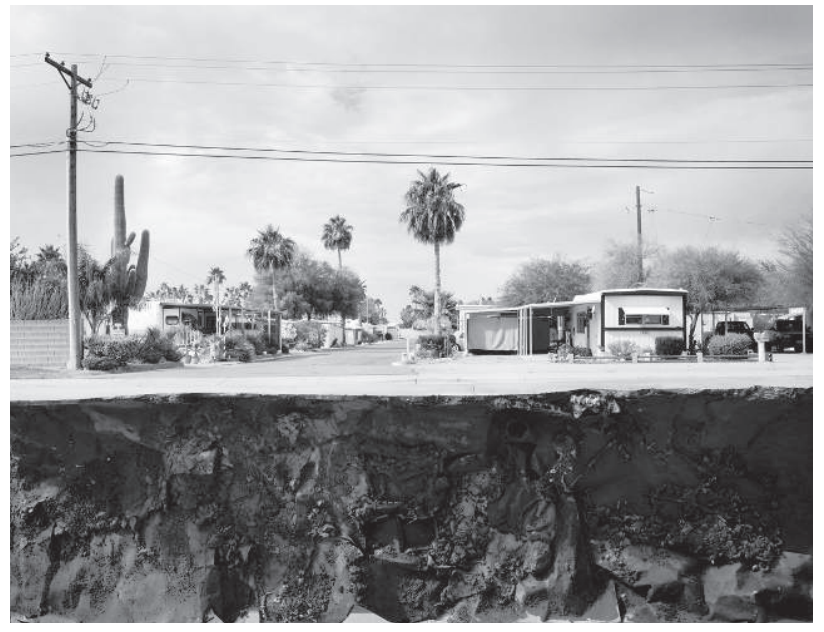

5/ Taiyo Onorato \& Nico Krebs, Abyss, 2006 (c-print, $94 \times 120$ cm, (c) Taiyo Onorato \& Nico Krebs; Courtesy the artists and Galerie RaebervonStenglin, Zurich) 
reflétée dans l'historiographie (encore succincte) des deux jeunes artistes zurichois ${ }^{9}$.

La reconstitution de ce paysage américain onirique se fait donc avec beaucoup de minutie. Leur travail de montage conjugue des sources visuelles de natures diverses, utilisant autant des images sans référent précis qui s’imposent assez logiquement (fig. 2) que des résurgences de photographies bien réelles ayant contribué à forger l'image de l'Amérique, dont l'origine ne peut être identifiée que par un spectateur averti. La moitié inférieure de l'image Abyss (2006, fig. 5) par exemple, est composée d'une maquette de plus de 2 mètres de long, soigneusement reconstituée par les artistes et transportée pendant plusieurs semaines dans leur camionnette, jusqu'à ce qu'ils eurent trouvé le paysage adéquat dans lequel ils pouvaient l'insérer ${ }^{10}$. Ce bricolage farfelu possède une fonction précise, puisque l'image résultante renvoie explicitement à une image célèbre de la landscape photography américaine, After a Flash Flood, Rancho Mirage, California, July 1979 de Joel Sternfeld (fig. 6). La référence à l'une des figures centrales de la New Color Photography

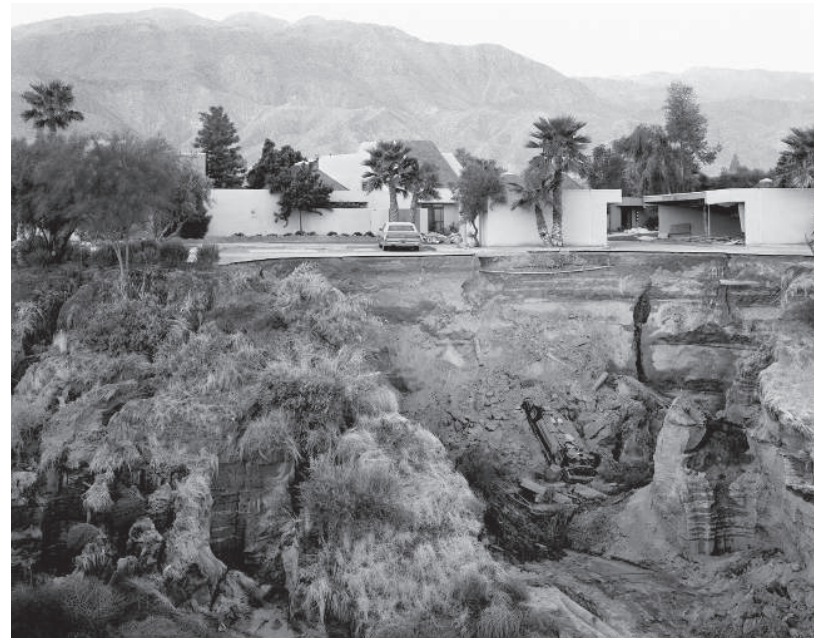

6/ Joel Sternfeld, After a Flash Flood, Rancho Mirage, California, July 1979, imprimé en 1987 (c-print, 40,6 ×50,8 cm, ( Joel Sternfeld; Courtesy of the artist and Luhring Augustine, New York)
9 Tyler Cobrun par exemple mentionne le caractère typiquement suisse du travail de Fischli \& Weiss et d'Onorato \& Krebs, (Tyler Cobrun, «Evolution. A new generation of photographers are literally taking their artworks into another dimension", Art Review, n 25 , septembre 2009, p. 94). L'association pour la distribution, la diffusion et la sauvegarde du cinéma expérimental Lightcone a pour sa part programmé Der Lauf Der Dinge (1987, $16 \mathrm{~mm}$, couleur, son, 29') de Fischli \& Weiss dans le cadre de l'exposition Ready (to be) made. Bas van Ader, Taiyo Onorato \& Nico Krebs (30 mai 25 août 2013), Le Bal, Paris.

10 Taiyo Onorato et Nico Krebs, conférence à l'Ecole cantonale d'art de Lausanne (écal), ro décembre 20I4. 


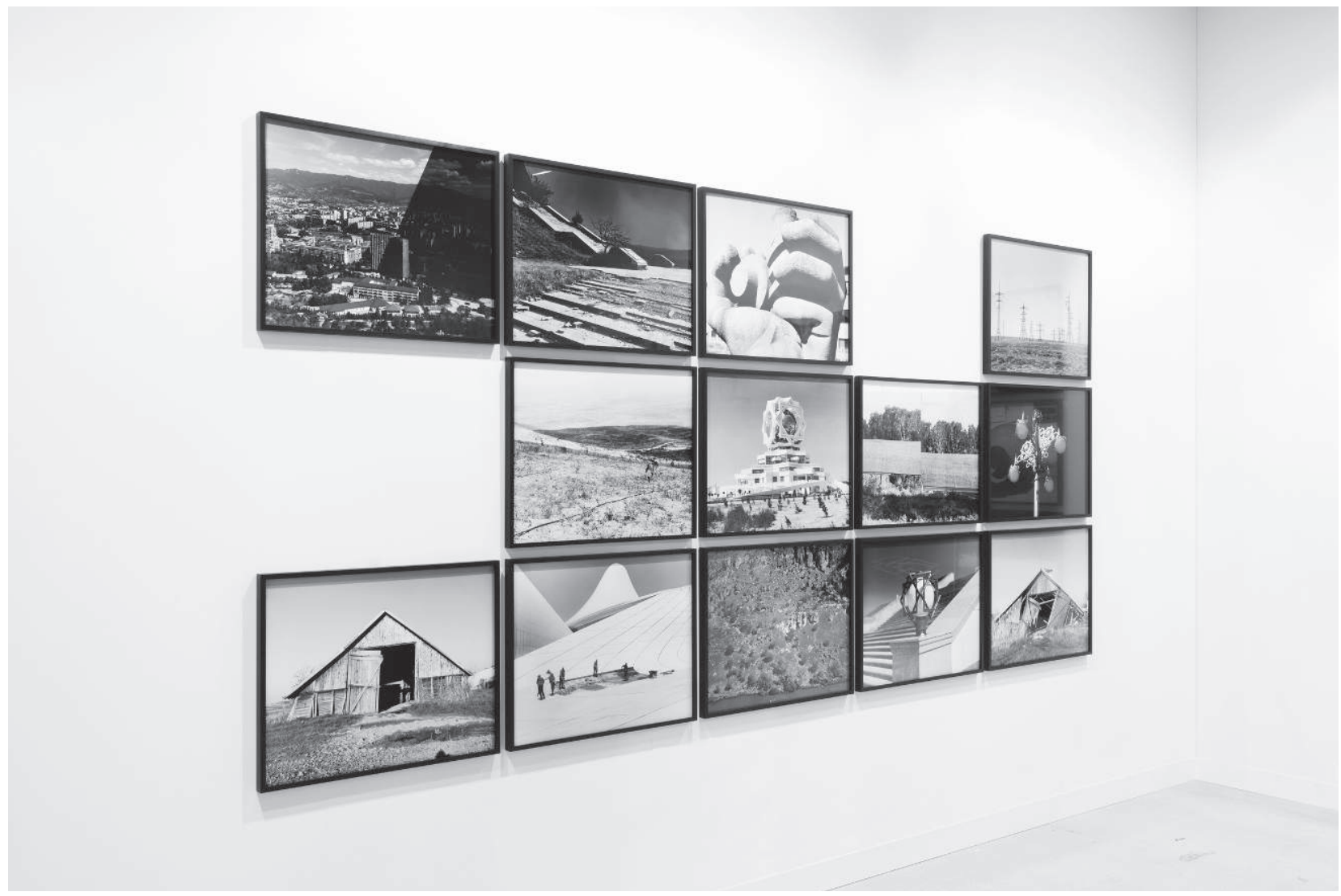

7/ Vue d'installation (photographie Gunnar Meier; Courtesy galerie RaebervonStenglin, Zurich)

américaine répond donc à un souci, mis en scène de manière élaborée, d'évoquer à des sources visuelles spécifiques. Entièrement assumées par les artistes, celles-ci complexifient le réseau de signification de la série du duo, articulant les représentations génériques de l’imaginaire collectif, des modèles spécifiques et leurs propres images fantastiques, créées de toutes pièces. Leurs «photomontages» occupent donc une position complexe, mobilisant les sources visuelles de cet imaginaire, suivant une conception collective, tout en proposant une lecture et interprétation personnelle de ces paysages. 
A la différence de The Great Unreal, Eurasia semble s'inscrire dans une relation au réel beaucoup plus directe. L'exposition bâloise se compose de trois éléments distincts. La trentaine de photographies en noir et blanc de taille moyenne $(50 \times 63 \mathrm{~cm})$, prises à la chambre grand format, provient du voyage de 2013. Elles sont arrangées géométriquement en grille et accrochées aux murs, composant deux tableaux photographiques de treize et seize images, chacun contenant deux emplacements vides (fig. 7). Les images se reflètent dans une structure en forme de prisme à base d'étoile recouverte d'un verre-miroir doré. Cette forme fait référence à l'emblème national turkmène, une étoile à huit branches (fig. 8), omniprésente dans l'architecture "turbo-capitaliste» "11 de la capitale Achgabat, sujet principal de la série exposée à Bâle. La surface dorée quant à elle renvoie au vocabulaire de façade de nombreuses constructions d'Asie centrale ${ }^{12}$, signifiant matériellement modernité et opulence. Finalement, l'installation contient en son cœur un écran montrant le transfert numérique d'un film i6mm couleur, Night of Colors (2013, I6mm transféré sur HD, 4 min 45 s), dévoilant essentiellement, en plans fixes nocturnes, les nombreuses décorations en néons clignotants qui ornent et soulignent l'architecture du chef-lieu turkmène.

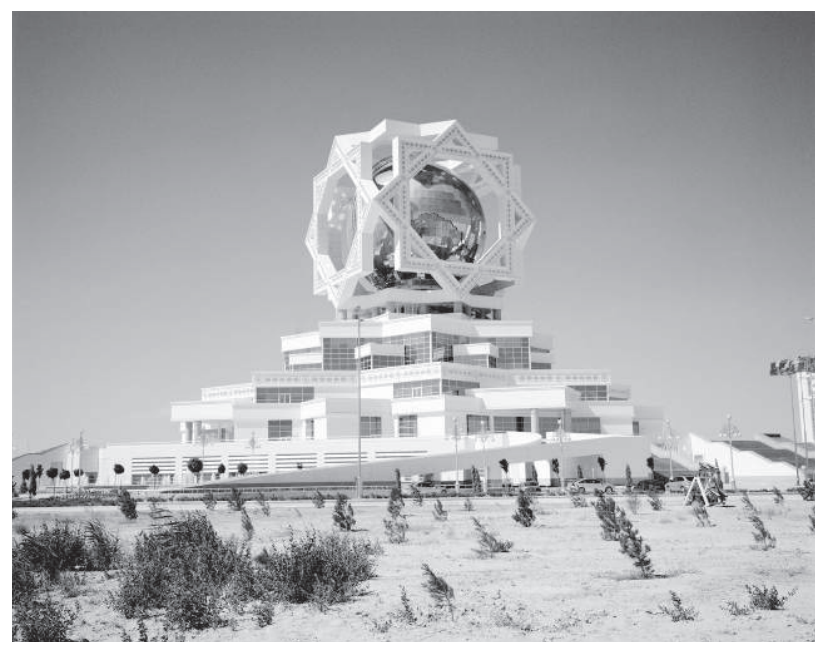

8/ Taiyo Onorato \& Nico Krebs, Wedding Palace, 2013 (tirage argentique, $50 \times 63 \mathrm{~cm}$, () Taiyo Onorato \& Nico Krebs; Courtesy the artists and Galerie RaebervonStenglin, Zurich) 

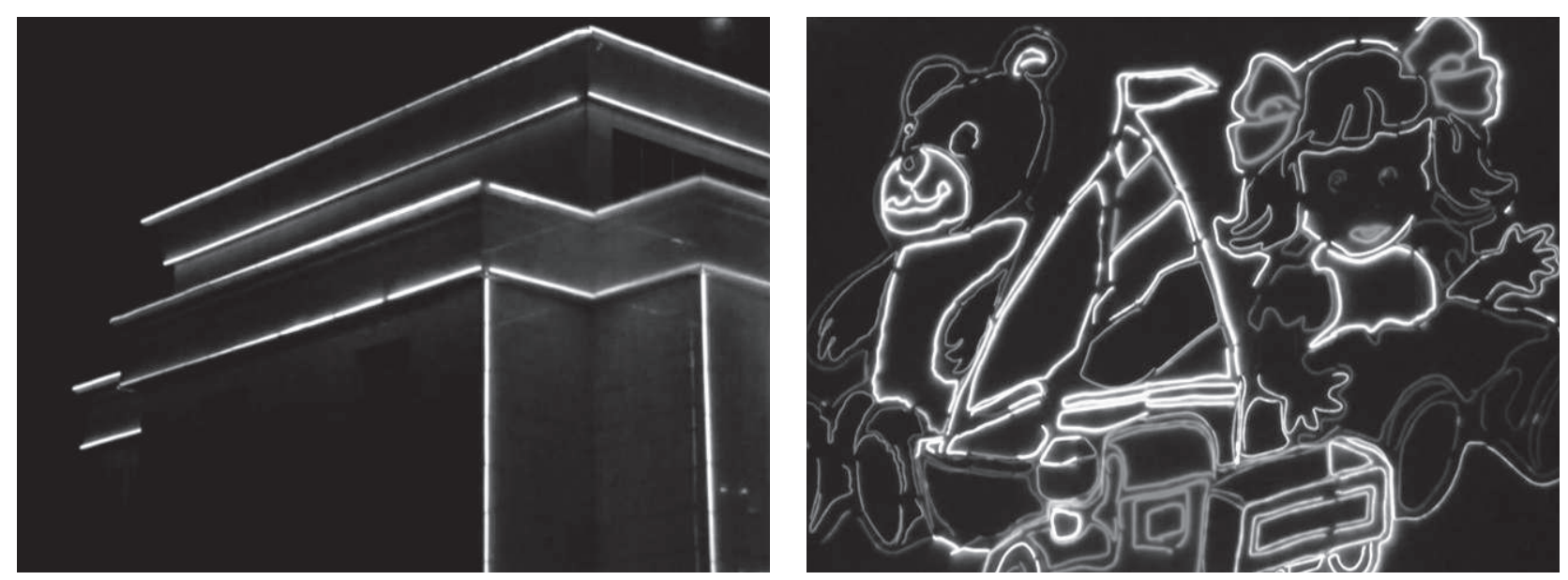

q et 10/ Taiyo Onorato \& Nico Krebs, Night of Colors, 2013, captures d'écran

13 Voir fig. 7, ligne du bas, deuxième image depuis la gauche.

14 Voir fig. 7, ligne du bas, première image depuis la gauche.

15 Voir fig. 7, ligne du bas, première image depuis la droite.

16 Voir fig. I, ligne du bas, première image depuis la gauche.
Ces trois éléments de l'installation articulent ainsi la production d'une identité visuelle par cette ancienne république soviétique, qui, grâce aux importants revenus pétroliers, a désormais acquis une place dans l'espace géopolitique mondial. Les façades de verre ou de marbre de la «ville blanche», l'étoile à huit branches omniprésente, les néons ou l'architecture globalisée - Zaha (2013) ${ }^{13}$ montre le Heydar Aliyev Cultural Centre à Baku (Azerbaïdjan), réalisé par Zaha Hadid, véritable star de l'architecture - sont autant de tentatives de ces anciens pays satellites de l'URSS de s'inscrire par l'image dans la géographie mondiale. Dans le travail d'Onorato et Krebs, l'analyse de cette production visuelle est contrebalancée par divers éléments qui révèlent les paradoxes de cette frénésie. En effet, la série et l'installation thématisent une opposition entre cette production de signes et le topos de la destruction, de la dégénérescence ou de l'absence. Ils opèrent, métaphoriquement d'abord, en confrontant par exemple deux images de la même étable, entière [Shed (Whole) $]^{14}$, puis détruite $[\text { Shed (Broken) }]^{15}$. Ils abordent également la question plus concrètement, en faisant dialoguer les symptômes de cette folie des grandeurs et sa déchéance quasi-immédiate: White City (2013) ${ }^{16}$ montre une ville moderne de nuit, baignée de lumière artificielle mais complètement vide, alors que Zaha expose les premières traces de déclin 


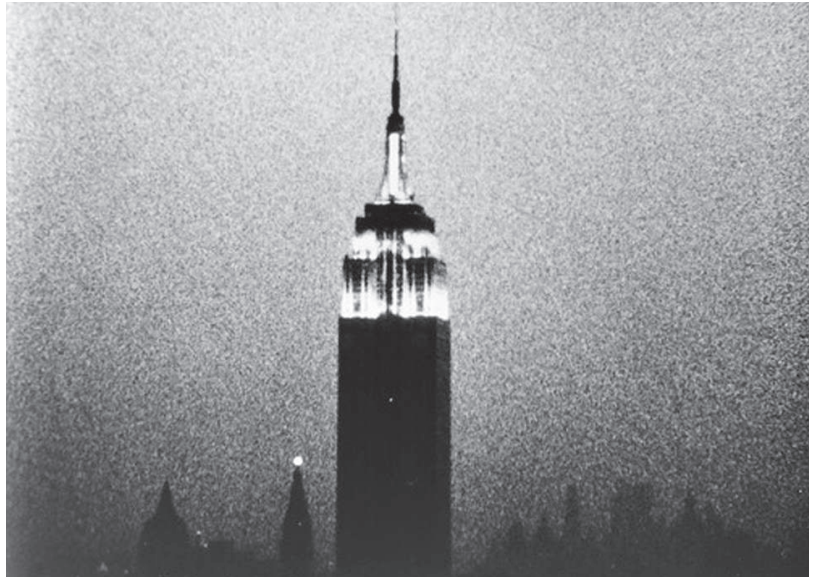

11/ Andy Warhol, Empire, $16 \mathrm{~mm}$, muet, 8 h 5 min, 16 images/seconde, 1964 (capture d'écran,

(c) Andy Warhol Foundation for the Visual Arts \& MoMA)

du bâtiment de l'architecte anglo-iraquienne, plus ou moins abandonné depuis sa présentation lors de l'Eurovision $2012^{17}$.

La sculpture dorée miroitante de l'exposition fait donc écho à cette volonté d'inscrire une nation dans le jeu géopolitique mondial à travers la construction de son image, ce que traduit presque explicitement le film qui y est projeté. Night of Colors, comme la totalité des films d'Onorato et Krebs ${ }^{18}$, enregistre en plans fixes des images très statiques, dans lesquels seulement quelques rares éléments bougent, par clignotement, incendie ou explosion. Dans le film Fire (I6mm, 3 min 44 s, 20I2), rattaché à la série Building Berlin ${ }^{19}$, on peut ainsi observer un échafaudage en bois, épousant en trompe-l'œil la forme d'un immeuble en arrière-plan, prendre feu et se consumer. Hormis les flammes et leur progression, l’image filmique est donc en tout point semblable à la série

17 Au XxI ${ }^{e}$ siècle, un certain type d'architecture, caractérisée par une reconduction de formes indépendante d'un contexte spécifique et légitimée par des architectes de renommée internationale, converge souvent avec la volonté d'asseoir un statut dans l'espace politique et économique mondial à travers le «city branding» et plus spécifiquement le «cultural branding» (construction de musées, d'opéras, de centres culturels, etc.). Voir par exemple Hans Ibelings,
Supermodernism. Architecture in the Age of Globalization, Rotterdam, NAi Publishers, 2003 au sujet d'une tendance acontextuelle en architecture et Graeme Evans, "Hard-Branding the Cultural City. From Prado to Prada», International Journal of Urban and Regional Research, vol. 27, $\mathrm{n}^{\circ}$ 2, juin 2003 et Kornelia Imesch Oechslin, «City, Urbanism, and the Art of a Brand: HafenCity Hamburg \& Saadiyat Island Cultural District», dans Kornelia Imesch Oechslin (éd.), Utopia and the Reality of Urbanism. La Chaux-de-Fonds-Chandigarh-Brasilia, Gollion, Infolio, 20I4 pour la question du branding culturel.

18 Les deux artistes ont élargi leur pratique à l'image en mouvement à partir du début des années 2010.

19 Voir par exemple l'ouvrage sur cette série Taiyo Onorato \& Nico Krebs. Raise the Bar, Paris, RVB Books/Le Bal, 2013. 
20 Nico Krebs, lors d'une conférence à l'Ecole cantonale d'art de Lausanne (écal), io décembre 20I4.

21 Comme par exemple les applications pour téléphone portable Cinemagram (Factyle) ou Motiongraph (Sony).

22 Le texte de l'architecte autrichien Adolf Loos «Ornament und Verbrechen» constitue sans doute le réquisitoire le plus célèbre du fonctionnalisme contre l'utilisation de l'ornement en architecture. Voir Adolf Loos, «Ornament und Verbrechen", Cahiers d'aujourd'hui, ${ }^{\circ}{ }^{5}$, I9I3.

23 Dans le cadre d'un projet de recherches avec leurs étudiants de la Yale Architecture School, Denise Scott Brown, Scott Venturi entreprirent avec l'aide de Robert Izenour l'étude de l'architecture vernaculaire de Las Vegas, abordant un nouveau type d'urbanité en rupture avec l'idéal fonctionnaliste du modernisme, à travers sa représentation visuelle. Signalétique, néons, ou éléments décoratifs y jouent un rôle essentiel, et l'utilisation par le Studio Yale de la photographie et du film comme forme analytique en découle directement. Par ailleurs, comme le note Martino Stierli, «le point de vue mobile et automobile» joue un rôle central dans l'expérience de la ville de l'Ouest américain d'aprèsguerre, ce qui impliquait une adaptation des moyens de représentations des formes urbaines. Voir Martino Stierli, «In Sequence: Cinematic Perception in Learning from Las Vegas ", Hunch, ${ }^{\circ}$ I2, 2009, pp. 76-77 (traduction par l'auteur) et Robert Venturi, Denise Scott Brown et Steven Izenour, Learning from Las Vegas, Cambridge, Mass./Londres, MIT Press, I972 (éd. française: L'Enseignement de Las Vegas, ou le symbolisme oublié de la forme architecturale, Bruxelles, Mardaga, 1987). photographique entreprise entre 2009 et 20I2, visant en même temps à questionner les fonctions illusionnistes du dispositif photographique et à réinvestir les nombreux terrains en friche au centre de Berlin ${ }^{20}$. Pour le spectateur contemporain, cette technique peut rappeler les logiciels utilisant le format GIF pour combiner une image photographique et une vidéo, en animant seulement une portion sélectionnée de l’image, le reste demeurant figé ${ }^{21}$. La référence formelle et conceptuelle de ce type d'images, dans l'histoire du cinéma, pourrait se situer dans des projets comme le film iconoclaste d'Andy Warhol Empire de i964 (fig. II), filmé par Jonas Mekas avec une Auricon I6mm. Les plans fixes d'une durée de huit heures sur l'Empire State Building filmé à la tombée de la nuit, «s'animent» grâce aux lumières qui s'allument au fur et à mesure que la nuit s'installe.

Dans le projet artistique du duo, ces films semblent donc s'inscrire dans la continuité d'œuvres photographiques préexistantes, comme c'est le cas pour Eurasia. Night of Colors prolonge la grandiloquence de l'architecture «diurne» par une ornementation lumineuse et propose ainsi un régime de signes différents des photographies, en se cantonnant à une expression nocturne du projet de communication visuelle kazakhe. Si celui-ci défie l'adage fonctionnaliste édicté par Adolf Loos ${ }^{22}$ - l'ornement est un crime -, cette iconographie rappelle l'intérêt pour ce type de formes visuelles chez Denise Scott-Brown, Robert Venturi et Steven Izenour dans le projet Learning from Las Vegas ${ }^{23}$. La réactualisation de cette iconographie se résume toutefois ici à un simple simulacre ornemental, les néons n’ayant ni référent, ni signification, ni réelle utilité. Parfois ils épousent simplement les formes des bâtiments afin de les rendre visibles de nuit, soulignant graphiquement leurs volumes architectoniques. D’autres fois encore il s'agit de «signes» dont la fonction sémantique est nulle, mais qui font visuellement référence, de manière lointaine, à des villes comme Las Vegas: cerises ou bouteilles stylisées rappellent par exemple l'univers du jeu ou du divertissement propre à cette ville du Nevada, mais à travers une représentation graphique, et non sa réalité physique. Mis à part la récurrence de l'étoile turkmène, il s'agit donc simplement d'une signalétique fantôme, dont la seule référence est le signe lui-même. En ce sens, Night of Colours insiste par l'absence de présence humaine et par une immobilité ostensible des plans, sur la dissociation entre un projet publicitaire, une manière d'ins- 
crire une ville dans le village global ${ }^{24}$ et ses très hypothétiques habitants - paramètre central du projet Learning from Las Vegas, qui revendiquait une architecture populaire axée sur la communication, subordonnée toutefois au principe de consommation ${ }^{25}$. Pour le visiteur occidental, le vocabulaire visuel de ces méta-signes énigmatiques constitue une originalité indéchiffrable et exotique qui, pour l'artiste visuel, devient un terreau d'expérimentation productif. En effet, si cette iconographie lumineuse ne possède pas de sens obvie, son vocabulaire familier permet un effet de contraste saisissant, donnant une familiarité déstabilisante à un monde, a priori, inconnu à ses yeux.

Le décryptage d'une image d'un paysage américain implique nécessairement, et de manière inconditionnelle, la mobilisation d'un équivalent mnémonique. The Great Unreal suggère la fonction révélatrice du travail d'Onorato et Krebs, articulé autour du rôle de l'inconscient visuel collectif, résidu idiomatique indéconstructible ${ }^{26}$. L'installation Eurasia déploie les mêmes mécanismes critiques mais révèle, par l'analyse de paysages peu familiers, la survivance d'un univers signalétique résiduel, oscillant entre la référence culturelle locale et l'image universelle et globalisée. L'appropriation (par les architectes kazakhes ou turkmènes) et la révélation (par les artistes) de ce système de signes hybrides produit donc une confrontation perçue par le spectateur occidental comme un univers visuel exotique familier, dialogue renforcé par la présence de divers médias - photographie, sculpture et films - dans l'installation. Le «territoire géopolitique sans image» est ainsi devenu un univers signalétique sans référence, une «coquille vide» ${ }^{27}$ dans l'espace géopolitique mondial.
24 Expression utilisée par le sociologue des médias Marshall McLuhan dans Understanding Media: The Extensions of Man (New York, McGraw-Hill, I964), afin de signifier les conséquences de la globalisation des réseaux médiatiques.

25 Cette position leur fut d'ailleurs vivement reprochée. Pour une synthèse des polémiques soulevées par l'ouvrage, voir en particulier Valéry Didelon, La Controverse Learning form Las Vegas, Wavre, Mardaga, 20II.

26 Jacques Derrida, L'Ecriture et la différence, Paris, Seuil, I967, pp. 310-3II.

27 Initié par Philippe Parreno et Pierre Huyghe, le projet collaboratif No Ghost Just a Shell résulte de l'achat d'un personnage manga nommé AnnLee à l'entreprise japonaise KWorks, qui produit du contenu pour l'industrie culturelle. AnnLee a ainsi été utilisée par à une multitude d'artistes (Liam Gillick, Dominique Gonzalez-Foerster, l'agence M/M, Rirkrit Tiravanija, ainsi que Huyghe et Parreno aux-même), projetant idées et concepts sous différentes formes dans cette «coquille vide». Voir en particulier No Ghost Just a Shell. Philippe Parreno et Pierre Huyghe, catalogue d'exposition (Kunsthalle Zurich/Institute of Visual Culture, Cambridge/Van Abbemuseum, Eindhoven, 2002-2003), Cologne, Walter König, 2003. 